\title{
Inadequação do consumo alimentar de nutrientes antioxidantes em nefropatas crônicos em hemodiálise
}

\section{Inadequate dietary intake of antioxidant nutrients for kidney disease patients on hemodialysis}

\begin{abstract}
Alana Flávia Fernandes dos Santos ${ }^{1}$. Christielle Félix Barroso ${ }^{2}$. Gueyhsa Nobre de Araújo ${ }^{3}$. Bruna Aparecida Melo Batista ${ }^{4}$. Isabelle Furtado Silva Cruz ${ }^{5}$. Denise Lima de Oliveira ${ }^{6}$. Carla Soraya Costa Maia ${ }^{7}$.

1 Graduada em Nutrição pela Universidade Estadual do Ceará (UECE). Nutricionista da Secretaria Municipal de Trabalho, Desenvolvimento Social e Combate à Fome, Prefeitura Municipal de Fortaleza, Ceará, Brasil. 2 Mestre em Nutrição e Saúde pela Universidade Estadual do Ceará (UECE), Professora do curso de Nutrição do Centro Universitário Estácio do Ceará, Fortaleza, Ceará, Brasil. 3 Graduada em Nutrição pela Universidade Estadual do Ceará (UECE). Nutricionista na empresa PRONEFRON, Fortaleza, Ceará, Brasil. 4 Graduada em Nutrição pela Universidade Federal do Rio Grande do Sul (UFRGS). Mestranda em Nutrição e Saúde pela Universidade Estadual do Ceará (UECE), Fortaleza, Ceará, Brasil 5 Mestre em Nutrição e Saúde pela Universidade Estadual do Ceará (UECE). Professora do curso de Nutrição das Faculdades Nordeste (FANOR), Fortaleza, Ceará, Brasil. 6 Mestre em Nutrição e Saúde pela Universidade Estadual do Ceará (UECE), Fortaleza, Ceará, Brasil. 7 Doutora em Nutrição Humana Aplicada pela Universidade de São Paulo (USP). Professora associada do curso de Nutrição da Universidade Estadual do Ceará (UECE), Fortaleza, Ceará, Brasil.
\end{abstract}

\section{RESUMO}

INTRODUÇÃO: o consumo de nutrientes antioxidantes por pacientes em hemodiálise é importante, pois a doença renal pode desencadear desequilíbrio oxidativo decorrente da intensa produção de espécies reativas, falhas nos sistemas antioxidantes e retenção do soluto oxidado. OBJETIVO: avaliar a adequação do consumo alimentar de nutrientes antioxidantes por pacientes renais crônicos em hemodiálise. MÉTODOS: o estudo foi desenvolvido com pacientes em tratamento de hemodiálise, afiliados a duas clínicas especializadas em Fortaleza, Ceará, Brasil. Os dados foram coletados por meio de três recordatórios de $24 \mathrm{~h}$ e tabulados no programa Excel $2007^{\circledR}$. Foram realizadas análises qualitativa e quantitativa da ingestão de selênio, zinco, vitamina A, vitamina $\mathrm{C}$ e vitamina E. O estudo incluiu 26 homens e 18 mulheres entre 19 e 83 anos de idade. RESULTADOS: encontraram-se médias de ingestão abaixo da EAR (Estimated Average Requeriment) de zinco, vitamina A, C e E e prevalências de risco de inadequação superior a $50 \%$ para todos os micronutrientes analisados, exceto o selênio. A ingestão de vitamina $\mathrm{E}$ foi inferior a EAR em 100\% dos pacientes. CONCLUSÃO: os pacientes renais crônicos em hemodiálise se encontravam com ingestão inadequada de micronutrientes antioxidantes, contribuindo para o aumento do estresse oxidativo.

Palavras-chave: Antioxidantes. Consumo de alimentos. Hemodiálise. Doença renal crônica.

\section{ABSTRACT}

INTRODUCTION: consumption of antioxidant nutrients for hemodialysis patients is important because chronic kidney disease can trigger oxidative imbalance due to the intense production of reactive species, failures in antioxidant systems and retention of oxidized solute. OBJECTIVE: to evaluate the inadequacy of food intake of antioxidant nutrients for kidney disease patients on hemodialysis. METHODS: the study was conducted with chronic kidney disease patients undergoing hemodialysis affiliated to two clinics in Fortaleza, Brazil. Data were collected using three 24-hour records and tabulated in Excel $2007^{\circledR}$. Analyzes were performed to assess qualitative and quantitative intake of selenium, zinc, vitamin A, vitamin C and vitamin $\mathrm{E}$. The study included 26 men and 18 women between 19 and 83 years. RESULTS: we found the mean intake below the Estimated Average Requeriment (EAR) for zinc, vitamin $\mathrm{A}, \mathrm{C}$ and $\mathrm{E}$ and prevalence of risk for inadequacy over $50 \%$ for all micronutrients analyzed, except selenium. The intake of vitamin E was less than EAR in 100\% of patients. CONCLUSION: the patients had inadequate intake of antioxidant micronutrients, which contributes to increased oxidative stress.

Keywords: Antioxidants. Food consumption. Hemodialysis. Chronic kidney disease.

Autor correspondente: Carla Soraya Costa Maia, Avenida Dr. Silas Munguba, 1700, Parangaba, Fortaleza, Ceará. CEP: 60740-903.Telefone: +55 85 3101-9644. E-mail: carla.maia@uece.br

Conflito de interesses: não há qualquer conflito de interesses por parte de qualquer um dos autores.

Recebido em: 09 Set 2016; Revisado em: 12 Out 2016; Aceito em: 22 Nov 2016. 


\section{INTRODUÇÃO}

A doença renal crônica (DRC) consiste em uma síndrome resultante de perda progressiva de filtração glomerular e capacidade de excreção renal, correspondendo a um ritmo de filtração glomerular inferior a $15 \mathrm{ml} / \mathrm{min} / 1,73 \mathrm{~m}^{2}$. Considerada um importante problema de saúde pública, demanda elevados investimentos financeiros: são gastos cerca de 1,4 bilhões de reais/ano com tratamentos de diálise e transplante renal no Brasil. $^{1}$

O tratamento da DRC terminal consiste em terapia renal substitutiva (TRS), que pode ser a hemodiálise (HD), a diálise peritoneal ambulatorial contínua ou automatizada e o transplante renal. ${ }^{1}$ Estima-se que, em 2015, cerca de 111.303 pacientes realizavam tratamento dialítico no país, e destes, $18,5 \%$ vieram a óbito. $^{2}$

A atividade pró-oxidante pode ocorrer no paciente renal crônico em hemodiálise por diversos mecanismos: inflamação crônica, idade avançada, síndrome urêmica, presença de diabetes, bioincompatibilidade com o sistema de membranas da HD, má nutrição, baixo consumo de nutrientes antioxidantes e perdas destes pelo processo dialítico. ${ }^{3,4}$

Selênio e zinco são minerais reconhecidos por seu papel antioxidante. O selênio faz parte do sítio ativo da enzima glutationa peroxidase, que atua reduzindo hidroperóxidos, como o peróxido de hidrogênio. ${ }^{5} \mathrm{O}$ zinco é componente da estrutura da superóxido dismutase, enzima catalisadora da reação de dismutação do ânion superóxido em peróxido de hidrogênio e oxigênio molecular. ${ }^{6}$

Dentre as vitaminas, têm seu papel antioxidante evidenciado as vitaminas A, C e E. A vitamina E tem atividade na membrana celular eliminando radicais livres e, assim como a vitamina $\mathrm{A}$, evita a peroxidação lipídica. ${ }^{7} \mathrm{~A}$ vitamina $\mathrm{C}$ possui atividade no meio aquoso da célula, prevenindo reações de oxidação, devido ao seu poder redutor. ${ }^{7}$

Dessa forma, a ingestão de nutrientes antioxidantes por esses pacientes é importante a fim de manter os sistemas de defesa antioxidantes do corpo em adequado funcionamento. Diante do exposto, este trabalho tem como objetivo avaliar o consumo de nutrientes antioxidantes por pacientes renais crônicos em tratamento de hemodiálise.

\section{MÉTODOS}

Trata-se de um estudo transversal, de abordagem quantitativa, realizado com pacientes adultos, com doença renal crônica em tratamento de hemodiálise, atendidos em duas clínicas especializadas em diálise em Fortaleza-CE. Não foram incluídos os pacientes portadores de doenças crônicas cujo diagnóstico encontrava-se nos prontuários médicos (diabetes, hipertensão, lúpus, câncer, artrite reumatoide e tireoidites) e pacientes com suplementação dos micronutrientes avaliados. O estudo foi aprovado no Comitê de Ética em Pesquisa da Universidade Estadual do Ceará com número de CAAE de 06048612.0.0000.5051 e todos os participantes foram esclarecidos sobre o objetivo do estudo e assinaram o Termo de Consentimento Livre e Esclarecido. A pesquisa foi realizada de acordo com os critérios estabelecidos pela Declaração de Helsinki com suas modificações. ${ }^{8}$

Os dados de consumo alimentar foram obtidos por meio da aplicação de três recordatórios de 24 horas (R24h), coletados em dois dias de semana não consecutivos e um dia de final de semana. A coleta foi realizada por entrevistadores treinados.

A composição da dieta em relação à energia, macronutrientes e a concentração de selênio, zinco e vitaminas A, C e E foram calculadas teoricamente pelo programa NUTWIN, versão 2.5 - CIS-EPM. ${ }^{9}$ O banco de dados do software foi alimentado com os dados da Base de Dados Nacional de Nutrientes para Padrão de Referência do Departamento de Agricultura dos Estados Unidos e de rótulos de alimentos, quando se tratavam de marcas específicas. Os dados foram tabulados através do programa Excel ${ }^{\circledR} 2007$.

Foi realizada a avaliação qualitativa e quantitativa da inadequação do consumo alimentar. A avaliação qualitativa foi feita para todos os micronutrientes analisados. Comparou-se a média de ingestão com os valores da EAR (Estimated Average Requeriment) e RDA (Recommended Dietary Allowance), ${ }^{10}$ considerando que, quando a ingestão média de um dado nutriente está abaixo da EAR, existe inadequação; quando está entre a EAR e a RDA, existe uma possível inadequação e quando está acima da RDA, assume-se que a ingestão é adequada. ${ }^{10}$

A avaliação quantitativa do consumo foi realizada individualmente, pois a amostra não ultrapassava 30 indivíduos do mesmo sexo e faixa etária. Para tanto, se utilizou a seguinte fórmula proposta pela US National Academy of Science Subcommittee on Criteria of Dietary Evaluation: ${ }^{10}$

$$
\begin{aligned}
& \mathrm{Z}=\mathrm{Mi}-\mathrm{EAR} / \sqrt{ }(\mathrm{Vn})^{2}+(\mathrm{Vi})^{2} / \mathrm{n} \\
& \text { Onde: } \\
& \mathrm{Mi}=\text { Média da ingestão } \\
& \mathrm{EAR}=\text { Estimated Average Requeriment } \\
& \mathrm{Vn}=\text { Variância da necessidade } \\
& \mathrm{Vi}=\text { Variância da ingestão intrapessoal } \\
& \mathrm{N}=\text { Número de dias de avaliação da ingestão }
\end{aligned}
$$

Os dados de variância intrapessoal foram obtidos do Continuing Survey of Food Intakes by Individuals. ${ }^{10}$ Para o selênio foram utilizados dados nacionais provenientes do estudo de Morimoto et al. ${ }^{11}$

A prevalência de risco de inadequação do consumo de nutrientes foi determinada através dos valores de distribuição normal.

Não foram analisadas as inadequações em casos de consumo superior a UL (Tolerable Upper Intake Level), para evitar desvios padrões muito elevados. 


\section{RESULTADOS}

Participaram do estudo 26 homens e 18 mulheres com recusa de apenas dois pacientes. A média de idade no grupo masculino foi de $51,11 \pm 18,7$ anos e no grupo feminino de 46,66 $\pm 17,3$ anos. Tempo de tratamento de menos de 59 meses para $48,94 \%$ dos pacientes, 60 - 120 meses para $25,53 \%$ e mais de 121 meses para $8,51 \%$ dos pacientes. As sessões de hemodiálise eram realizadas três vezes por semana com duração de quatros horas cada. As análises para vitamina $\mathrm{C}$ no grupo masculino contaram com 24 pacientes, e para vitamina $\mathrm{A}$ e $\mathrm{C}$ no grupo feminino com 17, pois foram retirados os valores acima da UL. Assim, três pacientes consumiam quantidades acima do máximo recomendado de vitamina $\mathrm{C}$ e uma paciente, de vitamina $\mathrm{A}$.

As médias de ingestão calórica e proteica no grupo feminino foram de 26,41 Kcal/Kg/dia e 1,14 g/Kg/dia, respectivamente. No grupo masculino encontrou-se ingestão média de 23,37 $\mathrm{Kcal} / \mathrm{Kg} /$ dia e $1,02 \mathrm{~g}$ de proteína/Kg/dia.
A avaliação qualitativa mostrou que a maioria dos pacientes, em ambos os grupos, possuía ingestão inferior a EAR para a maioria dos micronutrientes, exceto selênio. Para este, a maior parte dos pacientes apresentou ingestão acima da RDA, portanto, adequada.

A média de inadequação foi de $27,70 \%$ e de $55,55 \%$ na ingestão de selênio e zinco, respectivamente, no grupo feminino; e de $11,54 \%$ e $76,90 \%$, respectivamente, no grupo masculino. As vitaminas A, C e E apresentaram média de inadequação de $70,58 \%, 76,47 \%$ e $100 \%$, respectivamente, no grupo feminino; e de $88,46 \%, 83,33 \%$ e $100 \%$, respectivamente, no grupo masculino. A Figura 1 ilustra esses resultados.

A análise quantitativa demonstrou que a ingestão média de zinco e vitaminas A, C e E encontrava-se abaixo da EAR nos grupos feminino e masculino. Quanto ao selênio observou-se uma média de ingestão superior a RDA em ambos os grupos conforme demonstrado na Tabela 1. Assim, observa-se alta prevalência de risco de inadequação para esses micronutrientes.

Figura 1. Análise qualitativa da ingestão dos micronutrientes analisados nos grupos de pacientes em hemodiálise. Fortaleza, CE, Brasil, 2013.

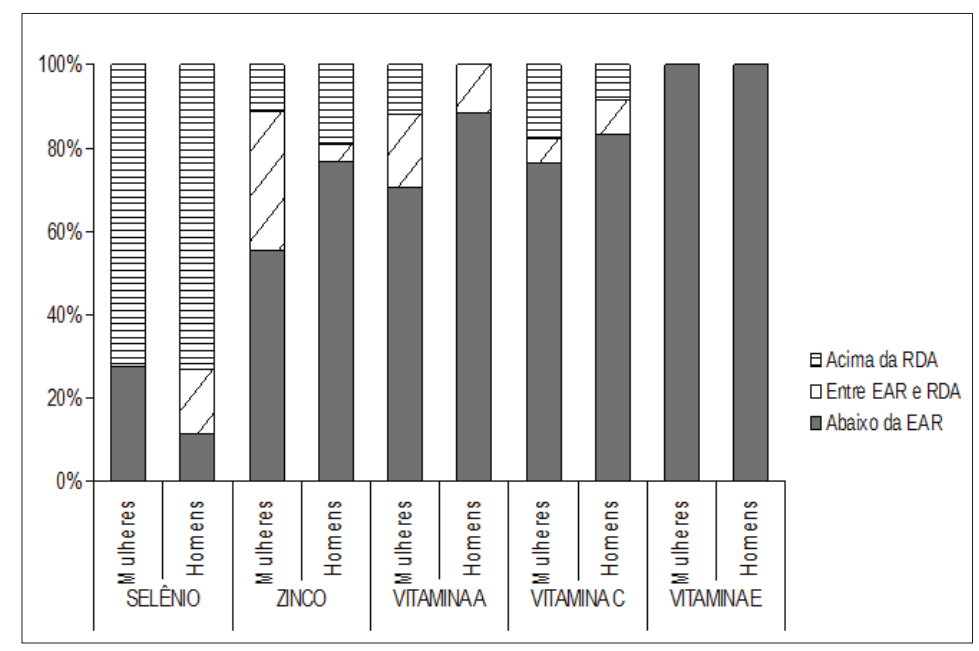

Tabela 1. Referência, média de ingestão dos micronutrientes e prevalência de risco de inadequação por sexo. Fortaleza, CE, Brasil, 2013.

\begin{tabular}{|c|c|c|c|c|}
\hline Nutrientes & EAR & RDA & Ingestão média & $\begin{array}{l}\text { Prevalência de Risco } \\
\text { de Inadequação }\end{array}$ \\
\hline \multicolumn{4}{|c|}{ Mulheres (>19 anos não gestantes e não lactantes) } & \\
\hline Selênio & $45 \mu \mathrm{g}$ & $55 \mu \mathrm{g}$ & $68,99 \mu \mathrm{g}$ & $22,2 \%$ \\
\hline Zinco & $6,8 \mathrm{mg}$ & $8,0 \mathrm{mg}$ & $6,32 \mathrm{mg}$ & $88,89 \%$ \\
\hline Vitamina A* & $500 \mu \mathrm{g}$ & $700 \mu \mathrm{g}$ & $463,06 \mu \mathrm{g}$ & $88,24 \%$ \\
\hline 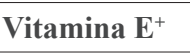 & $12 \mathrm{mg}$ & $15 \mathrm{mg}$ & $5,08 \mathrm{mg}$ & $100 \%$ \\
\hline Vitamina C & $60 \mathrm{mg}$ & $75 \mathrm{mg}$ & $35,91 \mathrm{mg}$ & $83,33 \%$ \\
\hline \multicolumn{4}{|c|}{ Homens ( $>19$ anos) } & \\
\hline Selênio & $45 \mu \mathrm{g}$ & $55 \mu \mathrm{g}$ & $80,62 \mu \mathrm{g}$ & $23,08 \%$ \\
\hline Zinco & $9,4 \mathrm{mg}$ & $11 \mathrm{mg}$ & $7,4 \mathrm{mg}$ & $80,77 \%$ \\
\hline Vitamina A* & $625 \mu \mathrm{g}$ & $900 \mu \mathrm{g}$ & $334,63 \mu \mathrm{g}$ & $96,15 \%$ \\
\hline${\text { Vitamina } E^{+}}^{+}$ & $12 \mathrm{mg}$ & $15 \mathrm{mg}$ & $4,32 \mathrm{mg}$ & $100 \%$ \\
\hline Vitamina C & $90 \mathrm{mg}$ & $68,4 \mathrm{mg}$ & $75 \mathrm{mg}$ & $88 \%$ \\
\hline
\end{tabular}

EAR: Estimated Average Requeriment; RDA: Recommended Dietary Allowance; *Equivalentes da atividade de retinol; $+\alpha$ - tocoferol 


\section{DISCUSSÃO}

Considerando a avaliação por sexo, houve maior prevalência de risco de inadequação na ingestão de vitamina $\mathrm{A}$, vitamina $\mathrm{C}$ e selênio no grupo masculino. Com relação à ingestão de zinco a prevalência de inadequação foi maior no grupo feminino. Isso se deve, provavelmente, a um maior consumo de carnes por parte dos homens em relação às mulheres, e a um maior consumo de vegetais por parte das mulheres, como observado nos recordatórios aplicados (dados não mostrados).

A prevalência de inadequação das análises qualitativa e quantitativa foi semelhante no caso do selênio e da vitamina E. Houve maiores diferenças no caso do zinco, vitamina A e vitamina $\mathrm{C}$, respectivamente. Vale ressaltar que as vitaminas $\mathrm{A}$, $\mathrm{C}$ e E não possuem distribuição normal, portanto, os dados da análise quantitativa não são totalmente confiáveis. No entanto, ambas as análises (qualitativa e quantitativa) demonstraram alta prevalência de risco de inadequação.

Os nutrientes antioxidantes deficientes na dieta contribuem para o aumento do estresse oxidativo, que na DRC pode favorecer o aumento da agregação plaquetária, leucopenia, vasoconstrição, aterosclerose, fibrose, apoptose, hipoalbuminemia, dislipidemia, redução da saturação de transferrina, resistência à eritropoietina, anemia, doenças cardiovasculares, infecções recorrentes, amiloidose, desnutrição, perda de massa muscular, envelhecimento acelerado, aumento da hospitalização e mortes. ${ }^{12}$

No tocante ao selênio, em pacientes com insuficiência renal crônica (IRC) observam-se níveis reduzidos do mineral, bem como atividade diminuída das glutationas. ${ }^{12}$ A ingestão recomendada pelas diretrizes da EBPG (European Best Practice Guidelines) para esses pacientes é de $55 \mu \mathrm{g} /$ dia, mesma recomendação para adultos saudáveis. ${ }^{13,14}$ A ingestão média encontrada no presente estudo, para ambos os sexos, foi superior a recomendação, podendo-se inferir que os sistemas antioxidantes dependentes de selênio nos pacientes estudados estejam adequados. No entanto, estudos de consumo alimentar referente ao selênio têm seu valor limitado considerando que este mineral tem diferente distribuição geoquímica. ${ }^{15}$ Assim as tabelas de composição de alimentos são limitadas nas concentrações desse mineral, bem como não elucidam quais os solos produtores dos alimentos ali registrados, inviabilizando uma análise fidedigna de consumo no Brasil, por exemplo.

Com relação à ingestão de zinco, as recomendações da EBPG são um pouco superiores às direcionadas a indivíduos saudáveis, sendo 8-12 mg para as mulheres e 10-15 mg para os homens. ${ }^{13,16}$ As médias encontradas neste estudo, para ambos, estão abaixo dessas recomendações, e a maioria dos participantes tem ingestão abaixo da EAR. A deficiência de zinco nesses pacientes pode contribuir não só para o aumento do estresse oxidativo, como também para neuropatia periférica, diminuição da resposta imunológica, intolerância à glicose e hiperlipidemia. ${ }^{17,18}$

$\mathrm{Na}$ IRC as concentrações de vitamina E dentro da célula estão diminuídas, sendo sua suplementação a terapia antioxidante mais utilizada nesse caso. ${ }^{12}$ As recomendações da EBPG para ingestão de vitamina $\mathrm{E}$ consistem na suplementação diária de 400-800 UI/dia $\left(268,46-536,91 \mathrm{mg}\right.$ de $\alpha$-tocoferol). ${ }^{13} \mathrm{O}$ estudo de Boaz et al..$^{19}$ analisou os efeitos da suplementação oral de $880 \mathrm{UI}$ de vitamina E/dia, o que equivale a 590,6 mg de $\alpha$-tocoferol, versus placebo em 196 pacientes em diálise e observou melhora no estresse oxidativo e redução na incidência de doenças cardiovasculares nos pacientes que receberam a vitamina E. ${ }^{19}$ No presente estudo, encontrou-se uma média de consumo de 5,08 $\mathrm{mg}$ de $\alpha$-tocoferol no grupo feminino e de 4,32 mg no grupo masculino, com todos os pacientes apresentando inadequação de consumo, demonstrando que provavelmente os sistemas antioxidantes relacionados à vitamina E estão debilitados nesses grupos.

As recomendações da EBPG para ingestão de vitamina A estão entre 700 e $900 \mu \mathrm{g}$, mesma recomendação para indivíduos saudáveis. ${ }^{13,16} \mathrm{~A}$ ingestão média de vitamina A neste estudo também se encontra abaixo da EAR, com a maioria dos pacientes sob alto risco de inadequação do consumo, principalmente no grupo masculino.

Com relação à vitamina $\mathrm{C}$, que também pode estar diminuída no paciente renal em virtude de sua perda durante a diálise, ${ }^{20}$ as médias de consumo encontradas tanto para homens como para mulheres foram inferiores a EAR, estando a maioria dos pacientes também com alto risco de inadequação do consumo.

No estudo de Araújo et al. ${ }^{21}$ realizado com os dados do Inquérito Nacional de Alimentação da Pesquisa de Orçamento Familiar (POF 2008-2009), que analisou a ingestão de macronutrientes e a inadequação de micronutrientes em 21.003 adultos brasileiros (52,5\% mulheres) entre 20 e 59 anos, observou-se uma menor prevalência de inadequação no consumo de selênio, zinco, vitamina A e vitamina $\mathrm{C}$ em relação ao observado no presente estudo. Com relação à vitamina $\mathrm{E}$, os valores foram semelhantes, demonstrando que a ingestão dessa vitamina no doente renal assemelha-se à ingestão da população geral, inferior às recomendações para indivíduos saudáveis. ${ }^{21}$

A diferença considerável na prevalência de inadequação do consumo de zinco encontrado neste estudo comparado à POF pode ser explicada pelo fato da dieta do paciente renal ser controlada em relação à quantidade de proteínas, e os alimentos fontes de proteína são também fontes do mineral. A prevalência de inadequação da vitamina $C$, diferente da população em geral, pode estar associada ao fato dos pacientes possuírem uma dieta restrita em potássio, o que exclui alguns alimentos fontes também de vitamina C. ${ }^{20}$

$\mathrm{O}$ estudo de Sahni et al..$^{22}$ que analisou a ingestão de zinco, vitamina $\mathrm{A}$ e vitamina $\mathrm{C}$ em pacientes com doença renal moderada e grave em pré-diálise, encontrou ingestão de 4,53 $\pm 1,89 \mathrm{mg}$ de zinco no grupo com doença renal moderada e de 5,42 $\pm 1,74 \mathrm{mg}$ no grupo com doença renal grave. Para a vitamina $A$, em equivalentes da atividade de retinol, foram encontrados 208,87 $\pm 90,09 \mu \mathrm{g}$ de ingestão no grupo com doença moderada e 190,31 $163,69 \mu \mathrm{g}$ de ingestão no grupo com doença grave. No caso da vitamina $\mathrm{C}$ encontrou-se ingestão de $21,95 \pm 11,56 \mathrm{mg}$ no grupo com doença moderada e de $17,09 \pm 10,6 \mathrm{mg}$ no grupo com doença grave. ${ }^{22}$ Os 
resultados sugerem que o tratamento conservador implica em maiores restrições dietéticas e consequentemente maiores inadequações alimentares, o que pode ser corroborado pelos melhores percentuais de inadequação de consumo dos pacientes em HD do presente estudo.

O estudo de Campo et al..$^{23}$ também encontrou alta prevalência de inadequação de micronutrientes. $\mathrm{O}$ estudo foi realizado com 73 pacientes em diálise peritoneal ambulatorial contínua e encontrou $85 \%$ destes com ingestão inferior às recomendações de zinco, e mais da metade com ingestão inferior às recomendações de vitaminas $\mathrm{A} \mathrm{e} \mathrm{C.}{ }^{23}$

Outro estudo, que avaliou o consumo alimentar de 291 pacientes em hemodiálise no Irã, encontrou ingestão média de $2 \pm 2 \mathrm{mg}$ de vitamina $\mathrm{E}$ e $99,7 \%$ dos pacientes ingerindo abaixo das recomendações; $35,5 \pm 29 \mathrm{mg}$ de vitamina C e $94 \%$ com

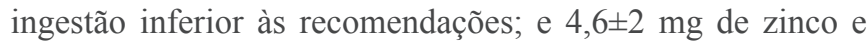
$100 \%$ com ingestão inferior as recomendações. ${ }^{24}$

No presente estudo foram encontrados valores de ingestão calórica abaixo das recomendações da EBPG, que recomenda de 30 a $40 \mathrm{Kcal} / \mathrm{Kg} /$ dia. ${ }^{13}$ Com relação a ingestão proteica, os valores médios encontrados estavam próximos às recomendações da EBPG, de $1,1 \mathrm{~g} / \mathrm{Kg} /$ dia. $^{13}$

É importante ressaltar que muitas vezes as carências dietéticas desses pacientes ocorrem em virtude da baixa ingestão

\section{REFERÊNCIAS}

1. Romão JE Jr. Doença renal crônica: definição, epidemiologia e classificação. J Bras Nefrol. 2004;26(3 Suppl 1):1-3.

2. Sociedade Brasileira de Nefrologia. Censo de diálise 2015 [Internet]. São Paulo; 2015 [acesso em: 2016 abr 17]. Disponível em: http://www.censo-sbn.org.br/censosAnteriores

3. McDonald CI, Fraser JF, Coombes JS, Fung YL. Oxidative stress during extracorporeal circulation. Eur J Cardiothorac Surg. 2014;46(6):937-43.

4. Stockler-Pinto MB, Lobo J, Moraes C, Leal VO, Farage NE, Rocha $\mathrm{AV}$, et al.. Effect of Brazil nut supplementation on plasma levels of selenium in hemodialysis patients: 12 months of follow-up. J Renal Nutr. 2012;22(4):434-9.

5. Burk RF, Hill KE. Regulation of selenium metabolism and transport. Annu Rev Nutr. 2015;35:109-34.

6. Jurowski K, Szewczyk B, Nowak G, Piekoszewski W. Biological consequences of zinc deficiency in the pathomechanisms of selected diseases. J Biol Inorg Chem. 2014;19:1069-79.

7. Koekkoek WA, van Zanten AR. Antioxidant vitamins and trace elements in critical illness. Nutr Clin Pract. 2016;31(4):457-74.

8. World Medical Association. World Medical Association Declaration of Helsinki. Ethical principles for medical research involving human subjects. Bull World Health Organ. 2001;79(4):373-4.

9. Universidade Federal do Estado de São Paulo. Nutwin: Programa de apoio à nutrição [CD-ROM]. Versão 1.5. São Paulo: UNIFESP; 2002. alimentar, ocasionada pela presença de anorexia, náuseas e/ou vômitos e alterações hormonais e metabólicas. ${ }^{25}$

De acordo com o estudo de Castillo e Gallegos, ${ }^{26}$ com o decorrer do tempo de tratamento renal substitutivo, o índice de massa corporal (IMC) e os valores de proteínas totais e albumina vão diminuindo, podendo demonstrar carências nutricionais proteico-calóricas. Kim et al. encontraram que o consumo energético insuficiente é a principal razão da ingestão reduzida de micronutrientes em pacientes em HD. ${ }^{25}$ Assim, antes de verificar as carências de micronutrientes, é necessário sanar esse problema.

Os dados encontrados nos trabalhos citados $^{22-25}$ corroboram com os do presente estudo no tocante ao alto risco de inadequação no consumo de micronutrientes antioxidantes por pacientes com doença renal.

\section{CONCLUSÕES}

Os pacientes apresentaram inadequada ingestão no consumo energético e de micronutrientes antioxidantes, o que contribui para quadro de desnutrição e desequilíbrio oxidativo, respectivamente.

Estudos correlacionando os micronutrientes e sua suplementação com biomarcadores nutricionais e de estresse oxidativo se fazem necessários junto aos pacientes nefropatas.

10. Institute of Medicine. Dietary reference intakes: applications in dietary assessment [Internet]. Washington; 2000 [acesso em: 2016 ago 20]. Disponível em: https://www.ncbi.nlm.nih.gov/books/ NBK222890/pdf/Bookshelf_NBK222890.pdf

11. Morimoto JM, Marchioni DM, Cesar CL, Fisberg RM. Variância Intrapessoal para ajuste da distribuição de nutrientes em estudos epidemiológicos. Rev Saúde Pública. 2011;45(3):621-5.

12. Prakash R, Singapalli T, Gokulnath. Review of oxidative stress in relevance to uremia. Clinical Queries Nephrology. 2012;1(3):215-21.

13. Fouque D, Vennegoor M, ter Wee P, Wanner C, Basci A, Canaud $B$, et al. EBPG guideline on nutrition. Nephrol Dial Transplant. 2007;22(Suppl 2):ii45-87.

14. Institute of Medicine. Dietary reference intakes for vitamin C, vitamin E, Selenium, and Carotenoids [Internet]. Washington; 2000 [acesso em 20 ago. 2016]. Disponível em: https://www.ncbi.nlm.nih. gov/books/NBK225483/pdf/Bookshelf_NBK225483.pdf

15. Shaltout AA, Castilho IN, Welz B, Carasek E, Martens IB, Martens A, et al. Method development and optimization for the determination of selenium in bean and soil samples using hydride generation electrothermal atomic absorption spectrometry. Talanta. 2011;85(3):1350-6.

16. Institute of Medicine. Dietary reference intakes for vitamin a, vitamin $\mathrm{k}$, arsenic, boron, chromium, copper, iodine, iron, manganese, molybdenum, nickel, silicon, vanadium, and zinc [Internet]. Washington; 2001 [acesso em 20 ago. 2016]. Disponível em: https://fnic.nal.usda. gov/sites/fnic.nal.usda.gov/files/uploads/vitamin_a_full_report.pdf 
17. Mazani M, Argani H, Rashtchizadeh N, Ghorbanihaghjo A, Hamdi A, Estiar MA, et al. Effects of zinc supplementation on antioxidant status and lipid peroxidation in hemodialysis patients. J Ren Nutr. 2013;23(3):180-4.

18. Wiggins KL. Renal Care: Resources and practical applications. [S.1]: American Dietetic Association; 2003. Seção 11: Vitamins and minerals in chronic kidney disease; p. 51-2.

19. Boaz M, Smetana S, Weinstein T, Matas Z, Gafter U, Iaina A, et al. Secondary prevention with antioxidants of cardiovascular disease in end stage renal disease (SPACE): randomised placebo controlled trial. Lancet. 2000;356(9237):1213-8.

20. Sirover WD, Liu Y, Logan A, Hunter K, Benz RL, Prasad D, et al. Plasma ascorbic acid concentrations in prevalent patients with end stage renal disease on hemodialysis. J Ren Nutr. 2015;25(3):292-300.

21. Araújo MC, Bezerra IN, Barbosa FS, Junger WL, Yokoo EM, Pereira RA, et al. Consumo de macronutrientes e ingestão inadequada de micronutrientes em adultos. Rev Saúde Pública. 2013;47(Supl 1):S177-89.
22. Sahni N, Gupta KL, Rana SV, Prasad R, Bhalla AK. Intake of antioxidants and their status in chronic kidney disease patients. J Ren Nutr. 2012;22(4):389-99.

23. Martín-del-Campo F, Batis-Ruvalcaba C, González-Espinoza L, Rojas-Campos E, Angel JR, Ruiz N, et al. Dietary micronutrient intake in peritoneal dialysis patients: Relationship with nutrition and inflammation status. Perit Dial Int. 2010;32(2):183-191.

24. As'Habi A, Tabibi H, Houshiar Rad A, Nozary Heshmati B, Mahdavi-Mazdeh M, Hedayati M. Dietary assessment of hemodialysis patients in Tehran, Iran. Hemodial Int. 2011;15(4):5307.

25. Kim H, Lim H, Choue R. A better diet quality is attributable to adequate energy intake in hemodialysis patients. Clin Nutr Res. 2015;4(1):46-55.

26. Castillo RF, Gallegos RF. Evolución del estado nutricional en pacientes en hemodiálisis durante 4 años de seguimiento. Arch Latinoam Nutr. 2011;61(4):376-81.

\section{Como citar:}

Santos AF, Barroso CF, Araújo GN, Batista BA, Cruz IF, Oliveira DL, et al. Inadequação do consumo alimentar de nutrientes antioxidantes em nefropatas crônicos em hemodiálise. Rev Med UFC. 2017 mai-ago;57(2):31-36. 